Sir,

\section{Purulent orbital cellulitis}

Regarding the case report by Redmill, Sandy and Rose, ${ }^{1}$ it should not be intuitively surprising that purulent orbital cellulitis resulted from a sub-Tenon's local anaesthetic given in the presence of active corneal ulceration. The authors do not state whether corneal scrapings were taken nor what the culture results of the scrapings were. The fact that they administered topical ofloxacin however suggests at least a clinical suspicion of active infection.

My personal experience suggests that sub-Tenon's or other invasive local anaesthetic are not necessary for the procedure of corneal gluing. This can be accomplished quite satisfactorily using topical anaesthesia in every case that I have dealt with. (A highly uncooperative patient would generally be unsuitable for gluing since they also presumably would be uncooperative following the procedure with a risk of eye rubbing.)

Perhaps therefore the main lessons to be drawn from this case report are firstly, that if invasive local anaesthesia is not necessary, it should not be used, and secondly, that it should be used with extreme caution in the presence of suspected or proven active infection of the external eye.

\section{References}

1 Redmill B, Sandy C, Rose GE. Orbital cellulitis following corneal gluing under sub-Tenon's local anaesthesia. Eye 2001; 15: 554-556.

\section{SJ Morgan}

Sunderland Eye Infirmary

Queen Alexandra Road

Sunderland SR2 9HP, UK

Correspondence: SJ Morgan FRCOphth

Tel: 01915656256

Fax: 01915699060

E-mail: stephen.morgan@chs.northy.nhs.uk.

Eye (2002) 16, 215. DOI: $10.1038 /$

sj/EYE/6700104
Sir,

Reply

We are grateful for the comments in this letter. Our patient presented with a full-thickness corneal melt associated with rheumatoid arthritis with no evidence of infection; therefore corneal scrapings were not taken. Topical ofloxacin was administered as a prophylactic measure in view of the use of a soft contact lens in the presence of an aqueous leak. We agree that topical anaesthesia is the most suitable technique for corneal gluing. Our report is of a previously unrecorded, but clinically significant complication of sub-Tenon's anaesthesia. This remains an invasive technique, which is not risk-free and should only be used if necessary.

B Redmill ${ }^{1}$, C Sandy ${ }^{2}$ and GE Rose ${ }^{2}$

${ }^{1}$ Opthalmology Dept

Lincoln County Hospital

Greetwell Road

Lincoln, LN2 5QY, UK

${ }^{2}$ Orbital Service

Moorfields Eye Hospital

London EC1V 2PD, UK

Correspondence: B Redmill

Tel: 01522573949

E-mail: brianredmill@mac.com

Eye (2002) 16, 215. DOI: $10.1038 /$

$\mathrm{sj} / \mathrm{EYE} / 6700092$

Sir,

'Lost' metallic vitrectomy port scleral plugs and MRI scanning

In pars plana vitrectomy the two active ports are sealed with either gold or silver plated scleral plugs

(Figure 1) when performing steps such as scleral

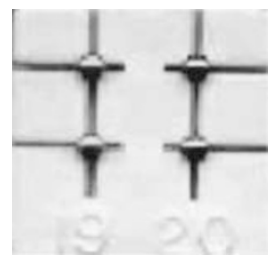

Figure 1 Gold and silver plated vitrectomy port plugs. 